\title{
A phase-only pulse shaper for multi-octave light sources
}

\author{
Vittorio Di Pietro, ${ }^{1,2}$ Simone Bux, ${ }^{2}$ Nicolas Forget ${ }^{2}$ and Aurélie Jullien ${ }^{1, *}$ \\ ${ }^{1}$ Institut de Physique de Nice, CNRS, Université Cote d'Azur, \\ UMR 7010, 1361 route des Lucioles, 06560 Valbonne, France \\ ${ }^{2}$ Fastlite, 165 route des Cistes, 06600 Antibes, France
}

\begin{abstract}
Continuous spectral phase shaping is demonstrated over a spectral bandwidth spanning from $540 \mathrm{~nm}$ to $2500 \mathrm{~nm}(450 \mathrm{THz})$ with a modulation dynamic large enough to shape single-cycle pulses or even transient electric fields in the near infrared.
\end{abstract}

The phase relationship between optical components plays a crucial role in controlling the electric field of broadband transient optical radiations [1] and a large number of devices have been proposed to shape the spectral phase: bulk media, prism, grating and grism pairs $[2,3]$, acousto-optic dispersive programmable filters (AOPDFs) [4], zero-dispersion lines [5], dispersive mirrors [6]. The growing development of light sources delivering pulses as short as a few optical cycles [7-12], either based on nonlinear post-compression or parametric amplification, has triggered the development of innovative pulse shaping or pulse compression devices, capable of handling bandwidths spanning over multiple optical octaves. Such spectral widths raise specific challenges: maximal spectral acceptance, minimal internal dispersion, high throughput are mandatory properties which tend to rule out most of the aforementioned techniques with the exception of thin wedges, thin plates and dispersive mirrors. To date, one of the most broadband and extreme pulse shaping device is an assembly of superoctave chirped mirrors used to compress and coherently combine up to four adjacent optical bandwidths ranging from the ultraviolet to the near-infrared [13]. Once manufactured, sets of dispersive mirrors are however characterized by a very small number of degrees of freedom and can hardly be compared to programmable shapers such AOPDFs or spatial light modulators (SLMs) inserted in zero-dispersion lines, in terms of shaping capability and flexibility.

SLMs usually consists in segmented elements, individually controlled by an electrical signal. Reflective SLMs are particularly suited as the zero-dispersion line may be folded while light propagates twice in the modulating layer, which, in turn, increases the dynamic range. Within the context of pulse shaping, two major classes of reflectives SLMs have been considered so far: deformable mirrors (such as MEMS [14] or thermally-deformable surfaces [15]) and liquid crystal (LC) on silicon (LCoS) [16]. In general, electrically-addressed devices provide independent control of the reflected light over a limited number of points, roughly equal to the number of actuators or electrode segments. Apart from inactive gaps between adjacent segments, a drawback of the LCoS technology

\footnotetext{
*aurelie.jullien@inphyni.cnrs.fr
}

is the presence of an unsegmented top electrode (usually ITO, typically transparent over $0.3-1 \mu \mathrm{m}$, with partial transmission up to $\simeq 2 \mu \mathrm{m}$ ) which strongly reduces the spectral bandwidth and damage threshold [17] of the device, by comparison with the LC mixture itself. This electrode is however mandatory as an oscillating electric field has to be maintained across the LC layer to control the average orientation of the molecular director.

Optical addressing solves the pixelisation issue and ensures arbitrary and continuous phase modulation $[18,19]$. In so-called light valve modulators, a biased photoconductive substrate replaces the segmented electrode and the voltage across the LC layer is locally controlled by an ancillary absorbed control beam, often referred as the "recording" beam, as opposed to the "readout" beam which is the (reflected) modulated beam. Here again, the top transparent electrode limits the spectral bandwidth and maximum power of the readout beam. Another strong limitation of conventional optical valves is the stringent requirement for an isolation layer to prevent cross-talk between the recording and readout beams.

Independently from the addressing technique, the thickness of the LC layer is $i 20 \mu \mathrm{m}$ in most SLMs, and results from a balance between the desired modulation range (e.g. maximum phase modulation), maximum control voltage, molecular disorder issues [20], and dynamic response time (typically about $1-100 \mathrm{~ms}$ for $10-90 \%$ rise and fall times). As the phase modulation range scales with the optical frequency, extending the spectral range towards the infrared tends to require thicker LC layers, which, in turn, constraints the electric addressing scheme.

In this paper, we propose an ultra-broadband reflective spatial light modulator, whose design is issued from our previous investigations on the use of thick cells of thermotropic nematic LCs [21-23]. The device, referred to as a Thermo-Optically-Addressed Spatial Light Modulator (TOA-SLM), is optically-addressed and circumvents most previous technical issues by eliminating the need for electrodes: the electrical addressing is replaced by an optically-induced control of the local temperature. The optical isolation between the recording and readout beam is performed by a single gold layer which endorses three additional functions: partial absorption of the recording beam, local heating of the LC, and the reflection of the readout beam. 
(a)



(b)

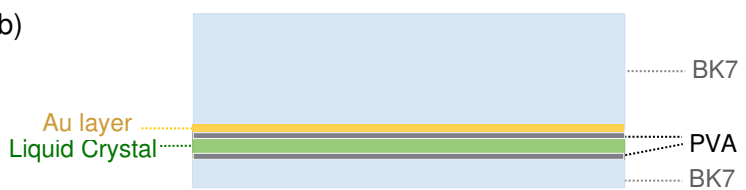

(c)

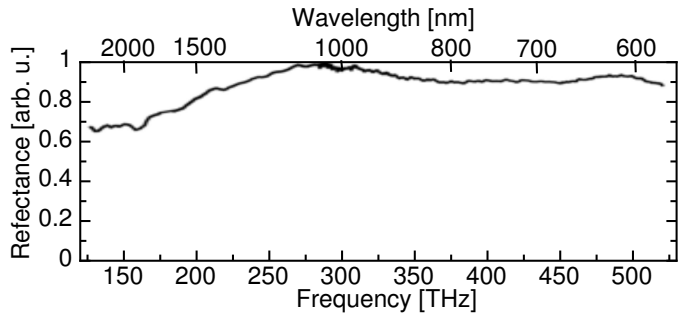

FIG. 1. Design of the TOA-SLM : illustration (a) and crosssection of the multi-layer structure (b). (c) Normalized spectral reflectivity.

In previous studies, a thermal-control of the refractive index of glass slabs have been exploited for phase shaping [24]. The thermotropicity of LCs is much higher than glass and, as a a matter of fact, thermo-optical valves have been widely investigated in the 80's in the context of displays [25]. The underlying physical mechanism was however different and relied on thermotropic LCs in the smectic mesophase with homeotropic organization. The local temperature was elevated via the absorption of a laser beam either by the LC or by an intermediate layer. The smectic-to-isotropic phase transition then provided amplitude modulation through the nucleation of static diffusion centers. The proposed TOA-SLM radically differs from the latter device by the modulated parameter (phase versus amplitude), the mesophase (nematic versus smectic), and the dynamic of the modulation (transient versus steady state).

The spectral bandwidth of the TOA-SLM is, in principle, delimited by the reflectivity of the gold layer and the transmission of LC and/or top substrate. Additionally, the modulation range exceeds one wave number, even at the longest wavelengths [23]. By inserting a TOA-SLM in a zero-dispersion line, we experimentally demonstrate arbitrary phase shaping over an ultra-broadband spectral bandwidth of $450 \mathrm{THz}(540-2500 \mathrm{~nm})$, this value being limited on the infrared side by the light source.

The TOA-SLM (FIG. 1a, b) is composed of: (i) a thick BK7 window $(6 \mathrm{~mm})$, coated on one side by a $100-\mathrm{nm}$



(b)

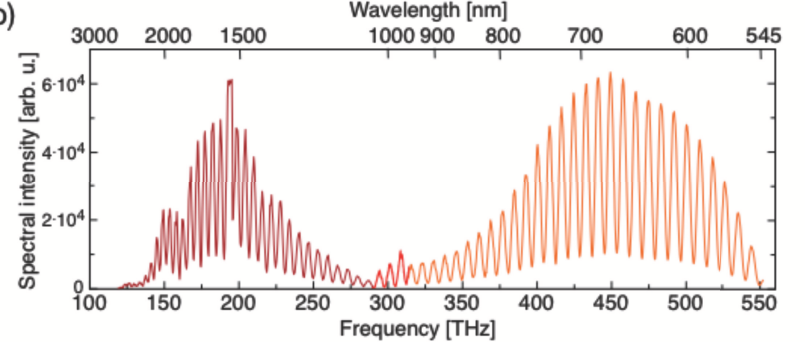

FIG. 2. a) Experimental setup: the ultra-broadband light source seeds a Michelson interferometer for referenced phase shaping measurement. The TOA-SLM is inserted in a 4f-line in a folded configuration. Yellow arrows indicate the laser polarization direction and the extraordinary axis of the LC layer. Three different spectrometers are used to collect the full spectral bandwidth (Avantes $550 \mathrm{~nm}-1000 \mathrm{~nm}$, Avantes $900 \mathrm{~nm}-1100 \mathrm{~nm}$, NIRQuest $900 \mathrm{~nm}-2600 \mathrm{~nm}$ ). b) Typical acquired spectra. Different color lines refer to the three different spectrometers.

gold layer and by an AR-coating at $465 \mathrm{~nm}$ on the other side, (ii) a thin BK7 window $(1 \mathrm{~mm})$ with a broadband AR-coated on one side and uncoated on the other side. A thin film of polyvinyl alcohol (PVA) is spin-coated on both the gold layer and the uncoated BK7 surface, and then rubbed to anchor the molecules in a plane parallel to the substrate. Finally, a layer of $50 \mu \mathrm{m}$ of the nematic mixture E7 (Merck) is inserted between the substrates, in contact with the PVA layers. The gold layer achieves simultaneously partial absorption ( $\simeq 60 \%$ absorption) of a recording beam at $465 \mathrm{~nm}$ and the reflection of the broadband readout light. The transverse dimensions are 25 $\mathrm{mm}$ per $25 \mathrm{~mm}$. As depicted in FIG. 1a, thermo-optical addressing is applied from the back of the TOA-SLM, through the thick substrate, with a $465 \mathrm{~nm}$ fiber-coupled laser diode $(600 \mathrm{~mW})$. The beam is partially absorbed by the gold layer, generates heat, and establishes a localized thermal gradient in the LC layer by diffusion. The LC being thermotropic and birefringent, both the ordinary and extraordinary optical refractive indices are locally modulated. Arbitrary modulation is achieved by spatially shaping the output of the $465 \mathrm{~nm}$ laser with digital light processing (DLP6500, Texas Instruments) and relay-imaging the DLP on the gold layer. 
The spectral reflectivity of the TOA-SLM is shown in FIG. 1c and spans from, at least, $120 \mathrm{THz}(2.5 \mu \mathrm{m})$ to $555 \mathrm{THz}(540 \mathrm{~nm})$ with $\mathrm{R}_{i} 80 \%$ between $200 \mathrm{THz}$ and $555 \mathrm{THz}$, and $\mathrm{R}_{\iota} 60 \%$ between $120 \mathrm{THz}$ and $200 \mathrm{THz}$. The measurement is here limited by the test source and should extend in the infrared to the transparency edge of the E7 mixture, that is $110 \mathrm{THz}(2700 \mathrm{~nm})$ [26].

The TOA-SLM is placed in the Fourier plane of a folded $4 f$-line: a BK7 glass wedge with an apex angle of $11^{\circ}$ disperses the optical frequency components in the horizontal plane, a $f=500 \mathrm{~mm}$ concave silver mirror images the glass wedge on the TOA-SLM in "2f-2f" configuration, and the TOA-SLM reflects the beam backward. The spatial extension of the spectrum is $\simeq 5 \mathrm{~mm}$ on the TOA-SLM (FIG. 3c), and the laser polarization direction matches that of the extraordinary axis of the LC. The resulting setup is inserted in a Michelson interferometer (FIG. 2a) and characterized by Fourier-Transform spectral interferometry. The test source is a super-continuum laser (ElectroVisir 250, Leukos) delivering $600 \mathrm{~mW}$ over the spectral bandwidth $120-550 \mathrm{THz}$. The reference arm of the interferometer includes compensation plates and a translation stage for tunable delay. The interference pattern is recorded by three different spectrometers and the corresponding spectra are stitched to cover the full spectral bandwidth (FIG. 2b). The relative spectral phase is extracted by numerical filtering in the Fourier space. The phase without any recording beam is considered as the reference phase and is subtracted to subsequent measurements. For this proof-of-concept experiment, the spectral phase of the wedge is balanced by the additional dispersion in the other arm of the interferometer and is thus not taken into account.

A first calibration is performed, without DLP patterning, by focusing the blue laser diode on the gold layer of the TOA-SLM and by laterally displacing the beam in order to address different parts of the dispersed spectrum. The beam size on the gold layer is $\sim 200 \mu \mathrm{m}$, and the intensity is kept below $50 \mathrm{~W} / \mathrm{cm}^{2}$. The measured spectral phase is plotted in FIG. 3a for 7 different positions of the blue focal spot. This experiment demonstrates the ability to continuously address the full spectral range $(>450 \mathrm{THz}, \sim 2000 \mathrm{~nm})$, with a maximum phase shift as high as $35 \mathrm{rad}$ in the visible range $(456 \mathrm{THz})$, and of $7 \mathrm{rad}$ for the infrared edge of the spectrum $(130 \mathrm{THz})$. The introduced phase scales inversely with $\lambda$, which is compatible with a constant change of the extraordinary index $\left(\Delta n_{e}=0.1\right)$ over the full spectral bandwidth. The spectral profile of the phase shift indicates a thermal gradient with a Lorentzian-like distribution. This shape could be reproduced by solving numerically the heat equation by taking into account the physical properties of the complete TOA-SLM structure and by assuming a steady state (e.g. thermal equilibrium). In practice, a few seconds are needed to reach the equilibrium. As can be seen in FIG. 3a, the extension of the thermal gradient limits the spatial resolution to several hundreds of micrometers,
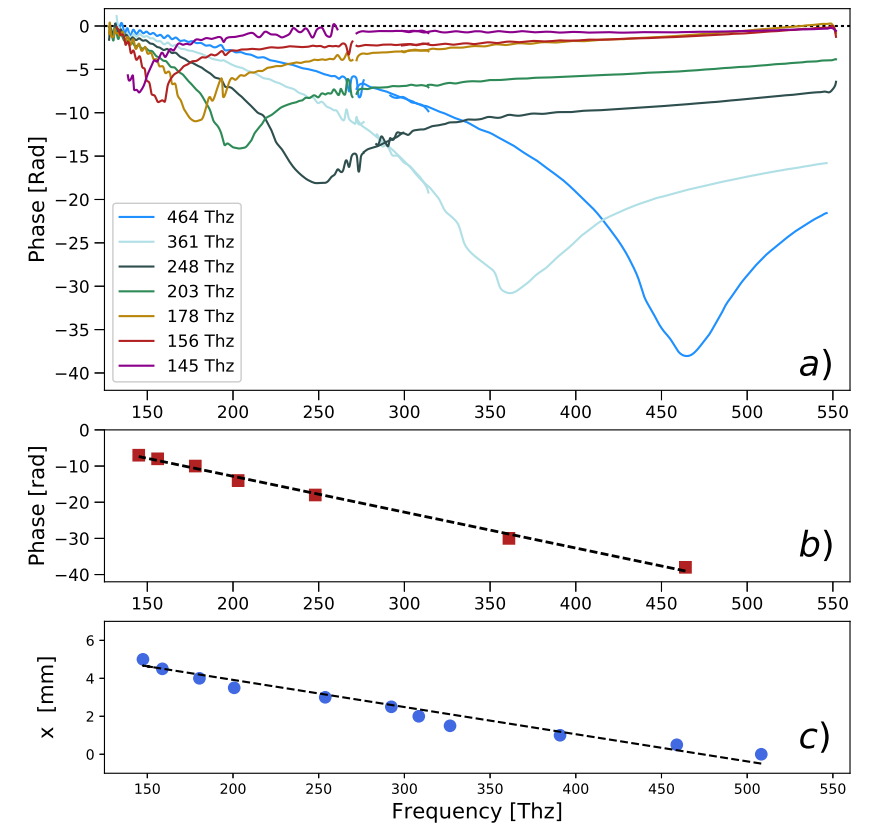

FIG. 3. Calibration of the TOA-SLM a) Spectrally-resolved phase shifts as a function of lateral positions of the recording beam. b) Maximum phase shift as function of the central frequency. (c) Calibration of the 4 f-line design: central frequency $\nu_{n}$ as a function of $x$.

thus limiting the spectral resolution. Here the resolution is sufficient to address slowly evolving phase orders, up to 5, after our calculations. The thermal evolution of the extraordinary index of E7 is written as [27]:

$$
n_{e}(T)=A-B T+\frac{2 \Delta n_{0}}{3}\left(1-\frac{T}{T_{c}}\right)^{\beta}
$$

where $T_{c}=331 K$ is the clearing point (nematicisotropic phase transition), and $A, B, \beta, \Delta n_{0}$ some fitting coefficients $\left(A=1.7546, B=5.36 * 10^{-4}, \beta=0.2391\right.$, $\left.\Delta n_{0}=0.3768\right)$.

Based on these properties, the performed calibration and the calculation of extension and shape of thermal gradient, the blue laser beam was the patterned with a linear combination of step-like functions, in order to apply a targeted spectral phase modulation : second order (e.g. quadratic) or third order (e.g. cubic) spectral phase (central frequency: $350 \mathrm{THz}$ ). A gallery of experimental examples of ultra-broadband phase shaping is shown in FIG. 4 a-d. Pure positive (resp. negative) quadratic phase with more than $8 \mathrm{rad}$ (resp $2.5 \mathrm{rad}$ ) (FIG. $4 \mathrm{a}, \mathrm{b})$ is recorded. The asymmetry between the positive and negative cases comes from the fact that it is more difficult to realize a temperature profile with positive curvature than a negative one, given the wide extension of Lorentzianlike distributions. In terms of phase coefficients, the total GDD span of $\pm 10 \mathrm{fs}^{2}$ is large enough to triple the pulse duration of a single-cycle Fourier-transform-limited pulse at $1 \mu \mathrm{m}(\simeq 3 \mathrm{fs})$. Pure positive (resp.negative) cubic phase 

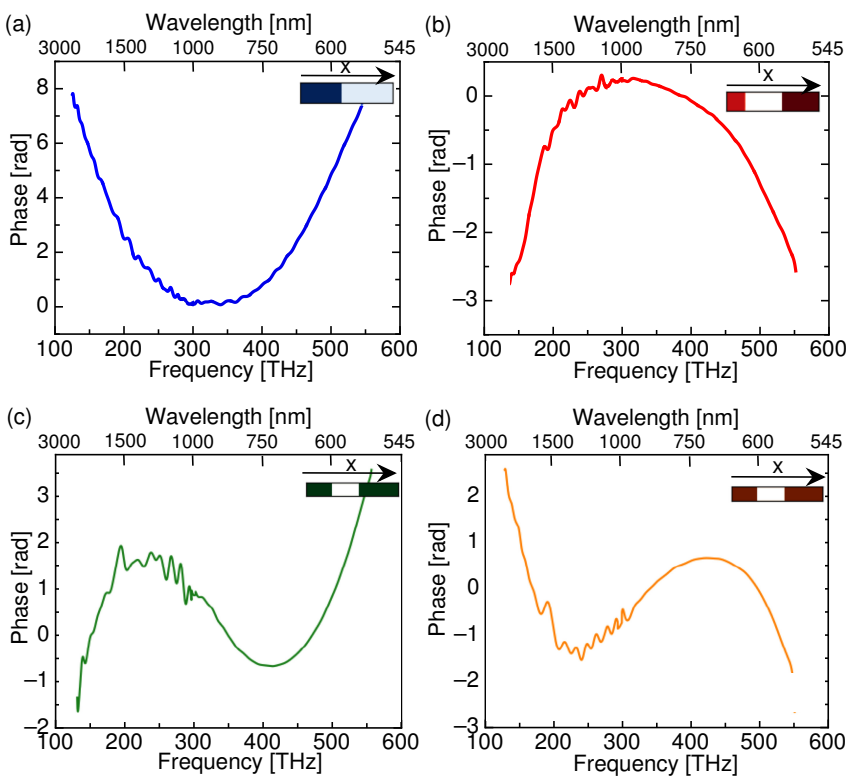

FIG. 4. Spectral phase modulation introduced by the $50 \mu \mathrm{m}$ thick TOA-SLM: a) positive GDD $\left(+8.2 \mathrm{fs}^{2}\right)$, (b) negative GDD $\left(-2.5 \mathrm{fs}^{2}\right),(\mathrm{c})$ negative TOD $\left(-13 \mathrm{fs}^{3}\right),(\mathrm{d})$ positive TOD $\left(13 \mathrm{fs}^{3}\right)$. In the top right corner figures the spatial pattern loaded on the DLP to reach the targeted phase modulation. The peak to valley temperature in the LC layer is respectively estimated to $12 \mathrm{~K}$ (a), $7 \mathrm{~K}(\mathrm{~b}), 4 \mathrm{~K}$ (c) and $15 \mathrm{~K}(\mathrm{~d})$.

is generated up to $\pm 5 \mathrm{rad}$, corresponding to third-order phase coefficient (TOD) of $\pm 13 \mathrm{fs}^{3}$ (FIG. 4 c,d). As the index variation $\Delta n_{e}$ is a function of the inner temperature, simple tuning of the phase coefficients is achieved by keeping a constant pattern on the DLP and by increasing/decreasing the average power of the blue diode. A power scan is plotted in FIG. 5 (a,b), demonstrating independent tunability of the GDD and TOD, over the full accessible range.

To assess the shaping scalability of TOA-SLM with the thickness of the LC layer, the same experiments were reproduced with a LC layer of $110 \mu \mathrm{m}$. Experimental results show that the shaping capability is more than doubled (FIG. 4 c,d). The chirp span reaches $\pm 25 \mathrm{fs}^{2}$ in the latter case. These values could still be increased with a more powerful recording laser since the induced temperature $\left(\sim 305 \mathrm{~K}\right.$, corresponding to $\left.\Delta n_{e}=0.04\right)$ is still well below the clearing point $\left(331 \mathrm{~K}, \Delta n_{e}=0.2\right)$ [27]. A recording laser with higher power together with a thick LC layer should enable to compensate for the wedge dispersion while preserving the TOA-SLM shaping capabilities. It must be noted that, although the current specifications are comparable with some electromagnetic deformable mirrors, the TOA-SLM is characterized by a larger versatility and a higher damage threshold (limited by the immersed gold surface). Combined to the almost unlimited aperture of LC devices, these features open the way to low-cost, large-aperture, programmable spectral phase shapers.
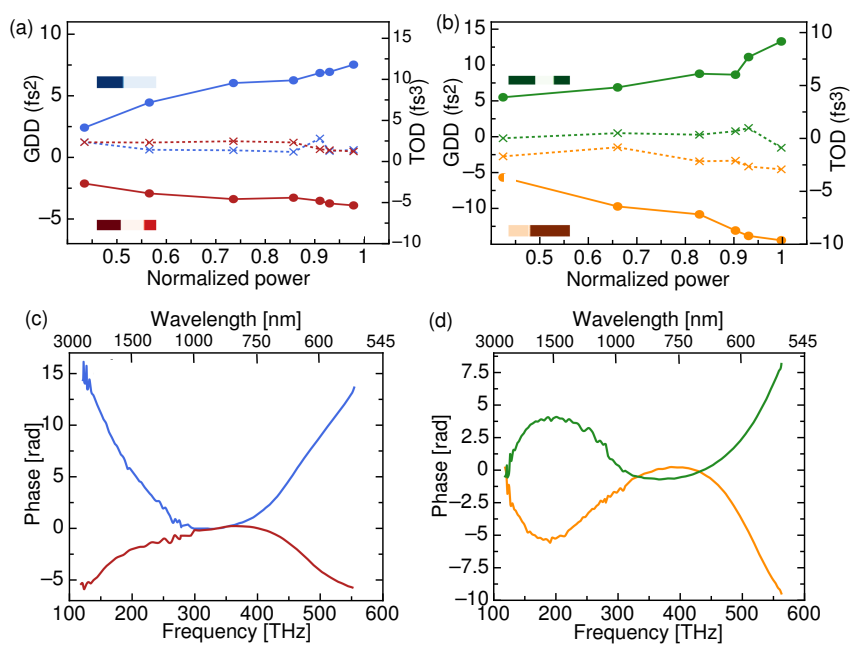

FIG. 5. (a,b) Spectral phase coefficients as a function of the normalized power of the blue light for the $50 \mu \mathrm{m}$-thick TOASLM: a) GDD (solid) and TOD (dotted) evolution for two different patterns (line and pattern colors match) b) TOD (solid) and GDD (dotted) evolution for two different patterns (line and pattern colors match) (c,d) Spectral phase modulation introduced by the $110 \mu \mathrm{m}$-thick TOA-SLM: c) positive GDD in blue $\left(+19 \mathrm{fs}^{2}\right)$, negative GDD phase in red $\left(-7 \mathrm{fs}^{2}\right)$, (d) negative TOD in green $\left(-19 \mathrm{fs}^{3}\right)$, and positive TOD in yellow $\left(18 \mathrm{fs}^{3}\right)$.

To conclude, we have developed a new phase shaper that overcomes the typical limitations of LC-SLM. Thermo-optical addressing enables to control the optical index of the LC without electrodes, making the device broadband and un-segmented. TOA-SLM is compatible with thick LC-layers (at least $110 \mu \mathrm{m}$ ) and the modulation range exceeds three wave number, even at the longest wavelengths. The optical dephasing can be easily controlled by tuning the average power of the recording laser beam. When inserted in a $4 \mathrm{f}$-line, pure quadratic or pure cubic phases are produced over a multi-octave spectral bandwidth $(>450 \mathrm{THz})$. The total introduced chirp span is large enough to provide a many-fold (up to 7) increase in duration of a single-cycle Fourier-transformlimited pulse at $\sim 1 \mu \mathrm{m}(3 \mathrm{fs})$. Forthcoming devices may include a silver layer deposited on the gold layer to further extend the spectral acceptance in the visible part of the spectrum. Shorter wavelengths might be addressed as well, as long as above the first absorption bands of carbon bonds (e.g. around $250 \mathrm{~nm}$ ). In that case, an aluminum reflective layer is required. Higher spatial resolution is expected from optimization of the multi-layers structure, currently under study. These results open the way to unprecedented temporal shaping capabilities of few-cycle and single-cycle pulses. 


\section{FUNDING}

Agence Nationale de la Recherche under the projects Labcom (ANR 15-LCV1-0002-01) and Investissements d'Avenir UCA-JEDI (ANR-15-IDEX-0001); European Union's HORIZON 2020 research program under the Marie Sklodowska-Curie grant (No. 641272). The publication reflects the author's view. The Research agency of the European Union is not responsible for any use that may be made of the information it contains.

[1] A. M. Weiner, "Ultrafast optical pulse shaping: a tutorial review," Opt. Comm. 184, 3669-3692 (2011).

[2] E. B. Treacy, "Optical pulse compression with diffraction gratings," IEEE J. Quant. Elec. 5, 45408 (1969).

[3] P. Tournois, C. R. Acad. Sci. 269, 455 (1969).

[4] P. Tournois, "Acousto-optic programmable dispersive filter for adaptative compensation of group delay time dispersion in laser systems," Opt. Comm. 140, 245 (1997).

[5] C. Froehly, B. Colombeau, and M. Vampouille, "Shaping and analysis of picosecond light pulses," Prog. Opt. 20, 65 (1983).

[6] V. Pervak, S. Naumov, F. Krausz, and A. Apolonski, "Chirped mirrors with low dispersion ripples," Opt. Exp. 15, (2007).

[7] A. Kessel and V. E. Leshchenko and O. Jahn and M. Krüger and A. Münzer and A. Schwarz and V. Pervak and M. Trubetskov and S. A. Trushin and F. Krausz and Z. Major and S. Karsch, "Relativistic few-cycle pulses with high contrast from picosecond-pumped OPCPA," Optica 5, 434 (2018).

[8] M. Ouillé, A. Vernier, F. Boehle, M. Bocoum, M. Lozano, J.P. Rousseau, Z. Cheng, D. Gustas, A. Blumenstein, P. Simon, S. Haessler, J. Faure, T. Nagy and R. LopezMartens, "Relativistic near-single-cycle optics at 1kHz," ArXiv1907:01239 (2019).

[9] Y.-G. Jeong, R. Piccoli, D. Ferachou, V. Cardin, M. Chini, S. Hädrich, J. Limpert, R. Morandotti, F. Légaré, B. E. Schmidt, L. Razzari, "Direct compression of 170-fs 50 -cycle pulses down to 1.5 cycles with $70 \%$ transmission," Scientific Reports 8, 11794 (2018).

[10] Q. Wang and J. Zhang and A. Kessel and N. Nagl and V. Pervak and O. Pronin and K. F. Mak, "Broadband mid-infrared coverage with few-cycle pulses via cascaded parametric processes," Opt. Lett. 44, 2566 (2019).

[11] M. Seidel, "A New Generation of High-Power, Waveform Controlled, Few-Cycle Light Sources," Spinger (2019).

[12] S. Vasilyev and I. Moskalev and V. Smolski and J. Peppers and M. Mirov and A. Muraviev and K. Vodopyanov and S. Mirov and V. Gapontsev, "Multi-octave vis- ible to long-wave IR femtosecond continuum generated in Cr:ZnS-GaSe tandem," Opt. Exp. 27, 16405 (2019).

[13] O. Razskazovskaya, F. Krausz and V. Pervak, "Multilayer coatings for femto- and attosecond technology," Optica 4, 129 (2017).

[14] M. Hacker and G. Stobrawa and R. Sauerbrey and T. Buckup and M. Motzkus and M. Wildenhain and A. Gehner, "Micromirror SLM for femtosecond pulse shaping in the ultraviolet" App. Phys. B. 76, 711 (2003).

[15] M. Kasprzack and B. Canuel and F. Cavalier and R. Day and E. Genin and J. Marque and D. Sentenac and G. Vajente, "Performance of a thermally deformable mirror for correction of low-order aberrations in laser beams," App. Opt. 52, 2909 (2013).

[16] A. M. Weiner and D. E. Leaird and J. S. Patel and J. R. Wullert, "Programmable femtosecond pulse shaping by use of a multielement liquid-crystal phase modulator," Opt. Lett. 15, 326 (1990).

[17] C. Dorrer and S. K.-H. Wei and P. Leung and M. Vargas and K. Wegman and J. Boulé and Z. Zhao and K. L. Marshall and S. H. Chen, "High-damage-threshold static laser beam shaping using optically patterned liquidcrystal devices," Opt. Lett. 36, 4035 (2011).

[18] C. Dorrer and F. Salin and F. Verluise and J. P. Huignard, "Programmable phase control of femtosecond pulses by use of a nonpixelated spatial light modulator," Opt. Lett. 23, 709 (1998).

[19] S. Residori, U. Bortolozzo and J. P. Huignard, "Liquid crystal light valves as optically addressed liquid crystal spatial light modulators: optical wave mixing and sensing applications," Liquid crystals review 6, 1-16 (2018).

[20] V. M. DiPietro, A. Jullien, U. Bortolozzo, N. Forget and S. Residori, 'Dynamical optical response of nematic liquid crystal cells through electrically driven Freedericksz transition : influence of the nematic layer thickness", Opt. Exp., 26, 10716 (2018).

[21] A. Jullien, U. Bortolozzo, S. Grabielle, J.-P. Huignard, N. Forget, and S. Residori, "Continuously tunable femtosecond delay-line based on liquid crystal cells," Opt. Exp. 24(13), 14483 (2016).

[22] A. Jullien, R. Pascal, U. Bortolozzo,N. Forget and S. Residori, "Liquid-crystal based Fourier-transform spectrometer applied to high-resolution hyperspectral imaging", Optica, 4, 400 (2017).

[23] V. M. di Pietro, A. Jullien, U. Bortolozzo, N. Forget, and S. Residori, "Thermally-induced nonlinear spatial shaping of infrared femtosecond pulses in nematic liquid crystals", Laser Phys. Lett. 16 015301, (2018).

[24] K. Osvay, K. Varju and G. Kurdi, "High order dispersion control for femtosecond CPA lasers", App. Phys. B 89, $565,(2007)$.

[25] A. G. Dewey, "Laser-addressed liquid crystal displays", Optical Engineering, 23, 230 (1984).

[26] Wu, "Infrared properties of nematic liquid crystals: an Overview". Optical Engineering, 26. 120-128 (1987).

[27] Li Jun, Electronic Theses and Dissertations (2005). 